\title{
Morphology and orientation of radio-loud Broad Absorption Line quasars
}

\author{
G. Bruni ${ }^{* 1,2,8}$, K.-H. Mack1 ${ }^{1}$, D. Dallacasa ${ }^{1,2}$, F.M. Montenegro Montes ${ }^{3}$, C.R. Benn ${ }^{4}$, \\ R. Carballo ${ }^{5}$, J.I. González-Serrano ${ }^{6}$, J. Holt ${ }^{7}$ and F. Jiménez-Luján ${ }^{4,6,8}$ \\ ${ }^{1}$ INAF - Istituto di Radioastronomia, Via P. Gobetti 101, I-40129 Bologna, Italy \\ 2 Dipartimento di Astronomia, Università di Bologna, Via Ranzani 1, I-40127 Bologna. Italy \\ ${ }^{3}$ European Southern Observatory, Alonso de Córdova 3107, Vitacura, Casilla 19001, Santiago, \\ Chile \\ ${ }^{4}$ Isaac Newton Group, Apartado 321, E-38700 Santa Cruz de La Palma, Spain \\ ${ }^{5}$ Dpto. de Matemática Aplicada y Ciencias de la Computación, Univ, de Cantabria, ETS \\ Ingenieros de Caminos Canales y Puertos, Avda. de los Castros s/n, E-39005 Santander, Spain \\ ${ }^{6}$ Instituto de Física de Cantabria (CSIC-Universidad de Cantabria), Avda. de los Castros s/n, \\ E-39005 Santander, Spain \\ ${ }^{7}$ Leiden Observatory, Leiden University, P O Box 9513, NL-2300 RA Leiden, The Netherlands \\ ${ }^{8}$ Dpto. de Física Moderna, Univ. de Cantabria, Avda de los Castros s/n, E-39005 Santander, \\ Spain
}

E-mail: brunieira.inaf.it

BAL QSOs are still a not-well understood class of objects. In the UV spectra they show Broad Absorption Lines (BALs) in the blue wings of the UV resonance lines, due to ionized gas with outflow velocities up to $0.2 \mathrm{c}$. Two different models have been proposed to explain this phenomenon: in the orientation model BAL-producing outflows should be present in all QSOs, but seen only when they intercept the observer's line of sight. In the evolutionary model BAL QSOs are young sources still expelling their dust cocoon.

We performed VLBI observations with both the EVN $(4.8 \mathrm{GHz})$ and VLBA $(4.8$ and $8.4 \mathrm{GHz})$ to map the pc-scale structure of the brightest radio-loud objects of our sample. A variety of morphologies and orientations have been found: 5 BAL QSOs in a total of 9 observed sources have a resolved structure, with a linear size $<1 \mathrm{kpc}$. In some cases the spectral index analysis of single components suggests a beamed emission toward the observer, in other cases a symmetric structure is evident from the map. From VLBI observations BAL QSOs do not seem to have a preferred orientation. Dimensions are typical of young GPS-CSS sources. This evidence could indicate an evolutionary scenario for the origin of this class of quasars.

10th European VLBI Network Symposium and EVN Users Meeting: VLBI and the new generation of radio arrays

September 20-24, 2010

Manchester, UK

*Speaker. 
Table 1: Sample of 9 radio-loud BAL QSOs studied in this paper. Column 4 is the redshift as measured from the SDSS, column 5 and 6 are flux densities measured with the VLA and presented in [2].

\begin{tabular}{ccccccc}
\hline Name & $\begin{array}{c}\text { RA } \\
(\mathrm{J} 2000)\end{array}$ & $\begin{array}{c}\text { DEC } \\
(\mathrm{J} 2000)\end{array}$ & $\mathrm{z}$ & \multicolumn{1}{c}{$\begin{array}{c}S_{4.8} \\
(\mathrm{mJy})\end{array}$} & \multicolumn{1}{c}{$\begin{array}{c}S_{8.4} \\
(\mathrm{mJy})\end{array}$} & Telescope \\
$(1)$ & \multicolumn{1}{c}{$(2)$} & $(3)$ & $(4)$ & \multicolumn{1}{c}{$(5)$} & \multicolumn{1}{c}{$(6)$} & \multicolumn{1}{c}{$(7)$} \\
\hline $0756+37$ & 075628.24 & +371455.6 & 2.515 & $226.2 \pm 2.0$ & $142.1 \pm 1.8$ & VLBA \\
$0816+48$ & 081618.99 & +482328.4 & 3.572 & $31.4 \pm 0.5$ & $19.5 \pm 0.5$ & EVN \\
$1005+48$ & 100515.98 & +480533.2 & 2.384 & $121.3 \pm 1.0$ & $83.6 \pm 1.1$ & VLBA \\
$1014+05$ & 101440.35 & +053712.6 & 2.013 & $34.5 \pm 0.6$ & $25.1 \pm 0.5$ & EVN \\
$1102+11$ & 110206.66 & +112104.9 & 2.350 & $39.8 \pm 0.8$ & $19.9 \pm 0.7$ & VLBA \\
$1237+47$ & 123717.44 & +470807.0 & 2.270 & $62.3 \pm 1.0$ & $61.6 \pm 1.6$ & VLBA \\
$1327+03$ & 132703.21 & +031311.2 & 2.827 & $79.5 \pm 1.7$ & $56.5 \pm 0.7$ & EVN \\
$1406+34$ & 140653.84 & +343337.3 & 2.565 & $312.8 \pm 3.1$ & $276.3 \pm 3.0$ & VLBA \\
$1603+30$ & 160354.15 & +300208.7 & 2.031 & $34.5 \pm 2.4$ & $26.9 \pm 0.6$ & EVN \\
\hline
\end{tabular}

\section{Introduction}

The nature and origin of BAL QSOs are still open issues in the framework of AGN morphology and evolution. The distinguishing characteristics of this class of object ( $\sim 15 \%$ of the entire QSO population) reside in their UV spectra, where Broad Absorption Lines (BALs) are present in the blue wings of the UV resonance lines, due to ionized gas with outflow velocities up to $0.2 \mathrm{c}$. Some evidence allowed several authors to propose an evolutionary scenario $([1,8])$, in which this phenomenon is due to the young age of these objects: the central AGN would still be expelling the enveloping dust cocoon. The duration of this phase with respect to the total life of a QSO would account for the $15 \%$ of BAL QSOs found in the entire population of QSOs. This view was supported by some experimental evidence $([5,2,7,4])$. An orientational model proposed by [3] foresees BAL outflows to be present in all QSOs, but only when these intercept the observer's line of sight the BALs are detected. In this case the dimension of the solid angle of the outflow would account for the percentage of BAL QSOs.

In previous works by our group $([5,2])$ no particular orientation has been found studying the spectral index of the synchrotron spectra, so we embarked on a VLBI project to test the inner structure of the QSO.

\section{Radio observations}

We used the VLBA for 6 sources in $\mathrm{C}$ and $\mathrm{X}$-band $(4.8 \mathrm{GHz}$ and $8.4 \mathrm{GHz})$ and the EVN in C-band for other 5 fainter sources.

0756+37: This source displays a nice double structure both in $\mathrm{C}$ and $\mathrm{X}$-bands. Component $\mathrm{A}$ has an integrated flux density of $59.2 \pm 5.9 \mathrm{mJy}$ in C-band and $48.8 \pm 4.8 \mathrm{mJy}$ in X-band, component B $88.2 \pm 8.8 \mathrm{mJy}$ in C-band and $72.2 \pm 7.2 \mathrm{mJy}$ in X-band. These values give a spectral index of -0.34 for A and -0.36 for B: both are flat spectrum sources. The separation between the centres of the two components is 2.1 mas that translates into a linear size of only $17 \mathrm{pc}$.

0816+48: This source shows a compact morphology, except for the first contour $(3-\sigma)$, where faint 
SE and NW extensions seem to be present. The flux density measured is $26.9 \pm 2.7 \mathrm{mJy}$. From the major and the minor axis of the Gaussian fit (6.8 and 4.7 mas) we can infer an upper limit of 51 and $35 \mathrm{pc}$, respectively.

1005+48: In this case no resolved structure has been detected, either in $\mathrm{C}$ or in $\mathrm{X}$-band, so just an upper limit for dimension can be given from a Gaussian fit of the source. Assuming the major and the minor axis of the Gaussian fit in X-band as an upper limit for dimensions (3.3 and 1.1 mas), we can put an upper limit to this source of $28 \mathrm{pc}$ and $9 \mathrm{pc}$, respectively. The flux densities at the two frequencies are $25.6 \pm 2.7 \mathrm{mJy}$ (C-band) and $20.0 \pm 2.1 \mathrm{mJy}$ (X-band). The spectral index is -0.44 : with a flat spectrum we can suppose this component to be a jet seen from the polar axis.

1014+05: This source presents a composed morphology: the central core is clearly visible as the brightest component (A), while a second (B) and a third (C) component are present toward the north. This could be interpreted as a core-jet structure, with an older faint component (C) with a flux density of $2.7 \pm 0.6 \mathrm{mJy}$ and a younger hotspot (B) with a more visible emission (7.4 $\pm 1.0 \mathrm{mJy}$ ), while the core (A) has a flux density of $10.3 \pm 1.1 \mathrm{mJy}$. The distance between $\mathrm{A}$ and $\mathrm{C}$ component is 26.6 mas, with a corresponding linear size of $228 \mathrm{pc}$.

1102+11: A symmetrical structure is clearly visible, with a strong component in the centre (A) and a fainter component toward NE (B). The first has a flux density of 28.1 $\pm 3.0 \mathrm{mJy}$ in C-band and $16.9 \pm 1.8 \mathrm{mJy}$ in X-band, while the second is present only in C-band with a flux density of $10.7 \pm 1.7 \mathrm{mJy}$. This allows one to calculate a spectral index of -0.9 for the A component, that could suggest an equatorial point of view for this source. The extension as calculated from the C-band image is 4.1 mas, thus $34 \mathrm{pc}$.

1237+47: This source shows a three-component structure, with the strongest in the centre (A) and the other two toward NW (B) and SE (C). The flux densities are $37.8 \pm 4.0 \mathrm{mJy}, 5.2 \pm 1.2 \mathrm{mJy}$ and $11.4 \pm 1.7 \mathrm{mJy}$ respectively in C-band, and $46.9 \pm 5.0 \mathrm{mJy}, 16.1 \pm 2.6 \mathrm{mJy}$ and $14.3 \pm 2.9 \mathrm{mJy}$ in $\mathrm{X}$-band. Spectral indices are 0.38 for A, 2.0 for B and 0.40 for the $\mathrm{C}$ component. The distance between the two most separated components is 4.2 mas, that is a linear size of $35 \mathrm{pc}$.

1327+03: This is a clear case of an unresolved source. The measured flux density is $80.6 \pm 8.0 \mathrm{mJy}$. The upper limit for the linear size is $48 \mathrm{pc}(6.0 \mathrm{mas})$ and $14 \mathrm{pc}(1.7 \mathrm{mas})$, respectively on major and minor axis, but the beam is considerably elliptical in this map so this is just an indicative value.

1406+34: This is another example of unresolved source, with an upper limit to linear size from the X-band image of $24 \mathrm{pc}$ on major axis ( 2.9 mas) and $11 \mathrm{pc}$ on minor axis (1.3 mas). The flux density in C-band is $81.2 \pm 11.4 \mathrm{mJy}$ while in X-band $195.1 \pm 20.6 \mathrm{mJy}$ : from these values we obtain an inverted spectral index equal to 1.57 .

1603+30: In this case a symmetric structure can be seen: this could be an example of a double-jet BAL QSO, dominated by the jet emission. Flux densities for the different components are (from east) $18.1 \pm 1.8 \mathrm{mJy}(\mathrm{A}), 8.8 \pm 1.0 \mathrm{mJy}(\mathrm{B})$ and $16.4 \pm 1.6 \mathrm{mJy}(\mathrm{C})$. Dimensions are $9.6 \mathrm{mas}$, linear size of $82 \mathrm{pc}$.

\section{Conclusions}

5 of 9 sources present a resolved structure, and various morphologies are visible. Double, core jet and symmetric structures have been found, so different orientations can be argued. Unresolved 


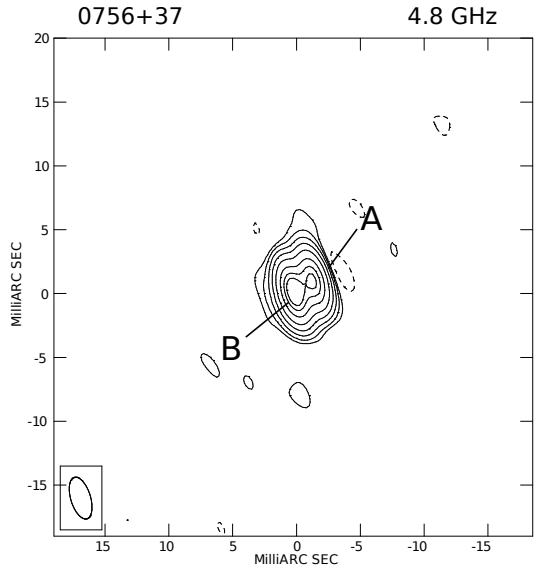

Levs $=0.97 \mathrm{mJy} *(-1,1,2,4,8,16,32,50,64)$

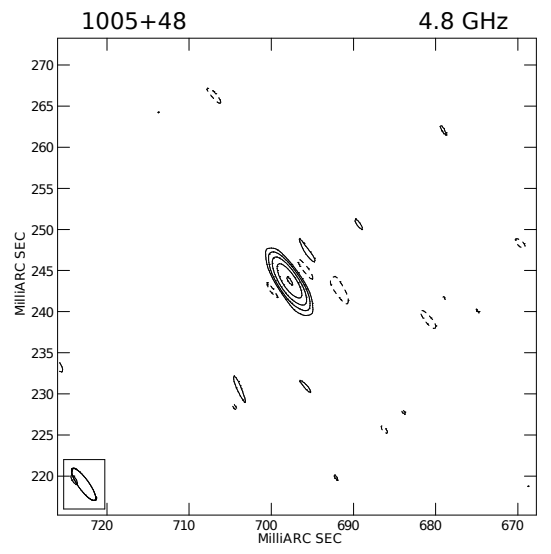

Levs $=1.27 \mathrm{mJy} *(-1,1,2,4,8,16,32)$

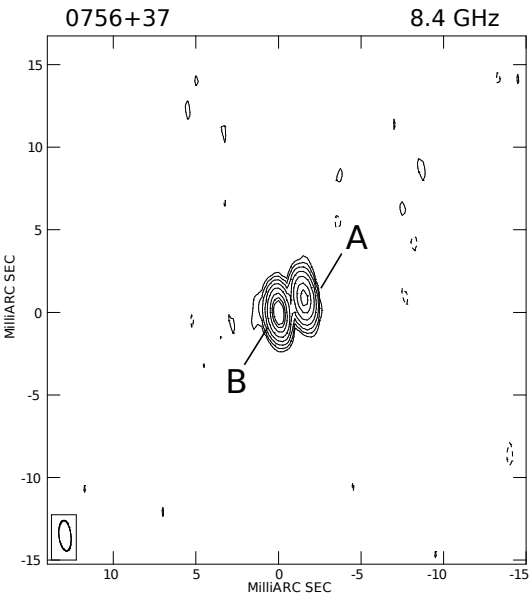

Levs $=0.67 \mathrm{mJy} *(-1,1,2,4,8,16,32,50,64)$

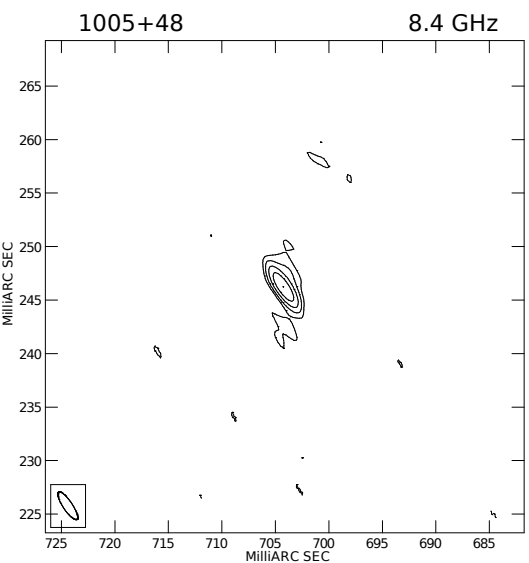

Levs $=0.96 \mathrm{mJy} *(-1,1,2,4,8,16,32)$

Figure 1: VLBA maps at $4.8 \mathrm{GHz}$ (left) and $8.4 \mathrm{GHz}$ (right) showing the pc-scale structure of BAL QSOS 0756+37 (top) and 1005+48 (bottom). The synthesised beam size is shown in the lower left corner of the map. Levels are 3- $\sigma$ multiples, according to the legend.

sources could be beamed jets towards the observer, like in case of $1005+48$, or extremely young sources, like in $1406+34$. Linear sizes are constrained under $1 \mathrm{kpc}$, so a classification like GPSCSS sources is possible. In this case a youth scenario can be supposed for BAL QSOs. The variety of orientations with respect to the observer and the variety of morphologies found imply a complex environment and seem to exclude a simple explanation of BAL QSOs within the orientation scenario proposed by [3].

\section{References}

[1] Briggs F. H., Turnsheck D. A., Wolfe M., The broad absorption lines in the spectrum of the QSO PKS 1157+014 - A possible link between broad absorption line QSOs, metal enrichment, and the formation of galaxies, ApJ, 287, 549 (1984)

[2] Bruni G., Mack K.-H., Salerno E., Montenegro-Montes F.M., Benn C., Carballo R., González-Serrano 

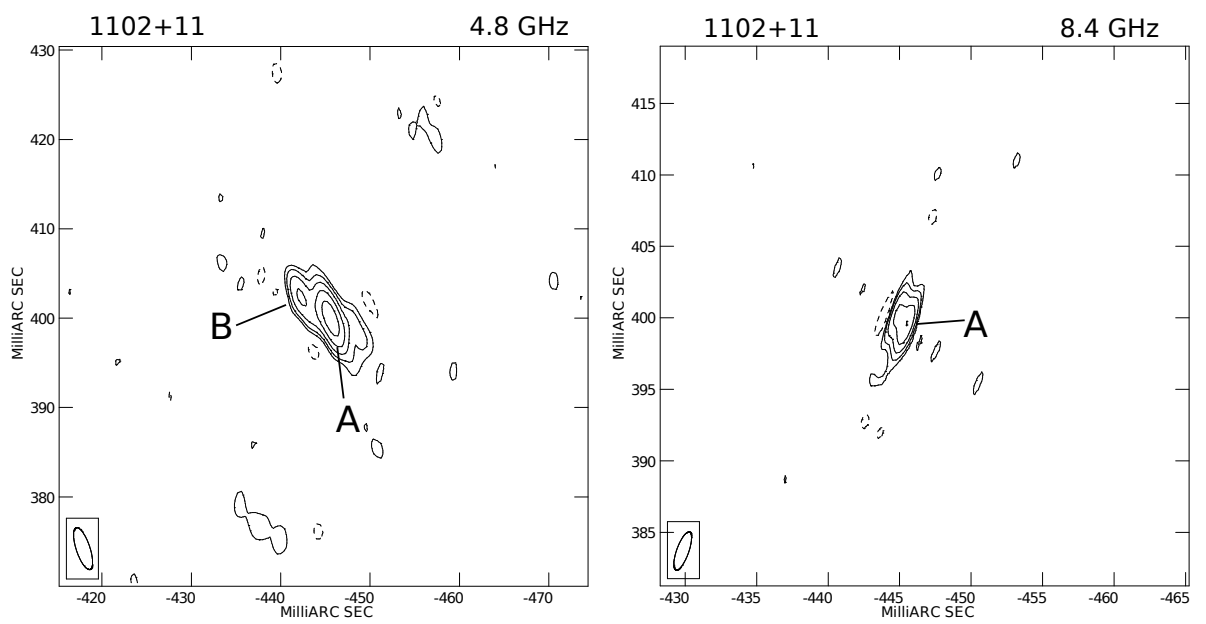

Levs $=0.81 \mathrm{mJy} *(-1,1,2,4,8,16,32)$
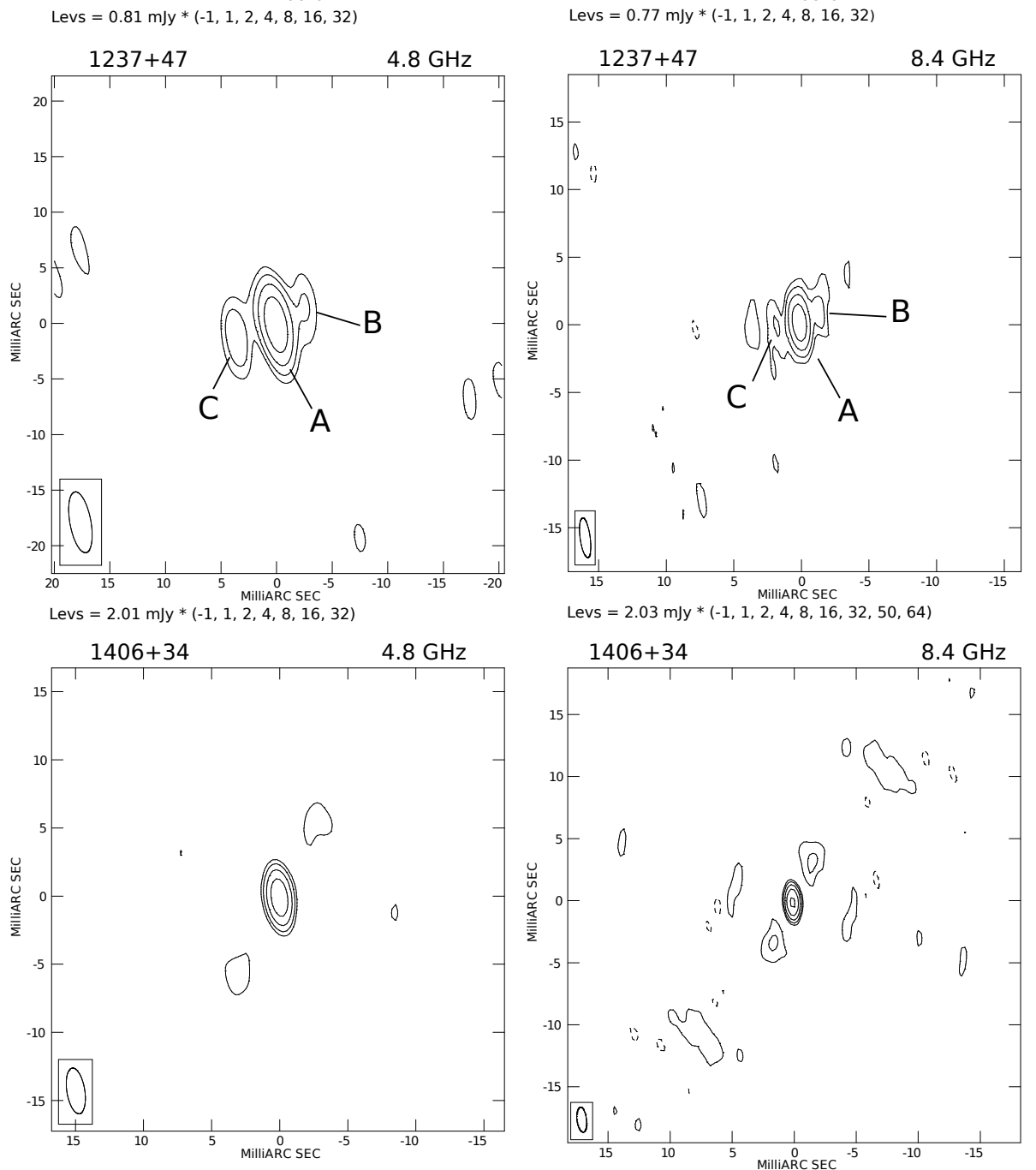

Levs $=15.69 \mathrm{mJy} *(-1,1,2,4,8,16,32,50,64)$

Levs $=0.85 \mathrm{mJy} *(-1,1,2,4,8,16,32,50,64)$

Figure 2: VLBA maps at $4.8 \mathrm{GHz}$ (left) and $8.4 \mathrm{GHz}$ (right) showing the pc-scale structure of BAL QSOs $1102+11$ (top), 1237+47 (centre) and 1406+34 (bottom). The synthesised beam size is shown in the lower left corner of the map. Levels are 3- $\sigma$ multiples, according to the legend. 

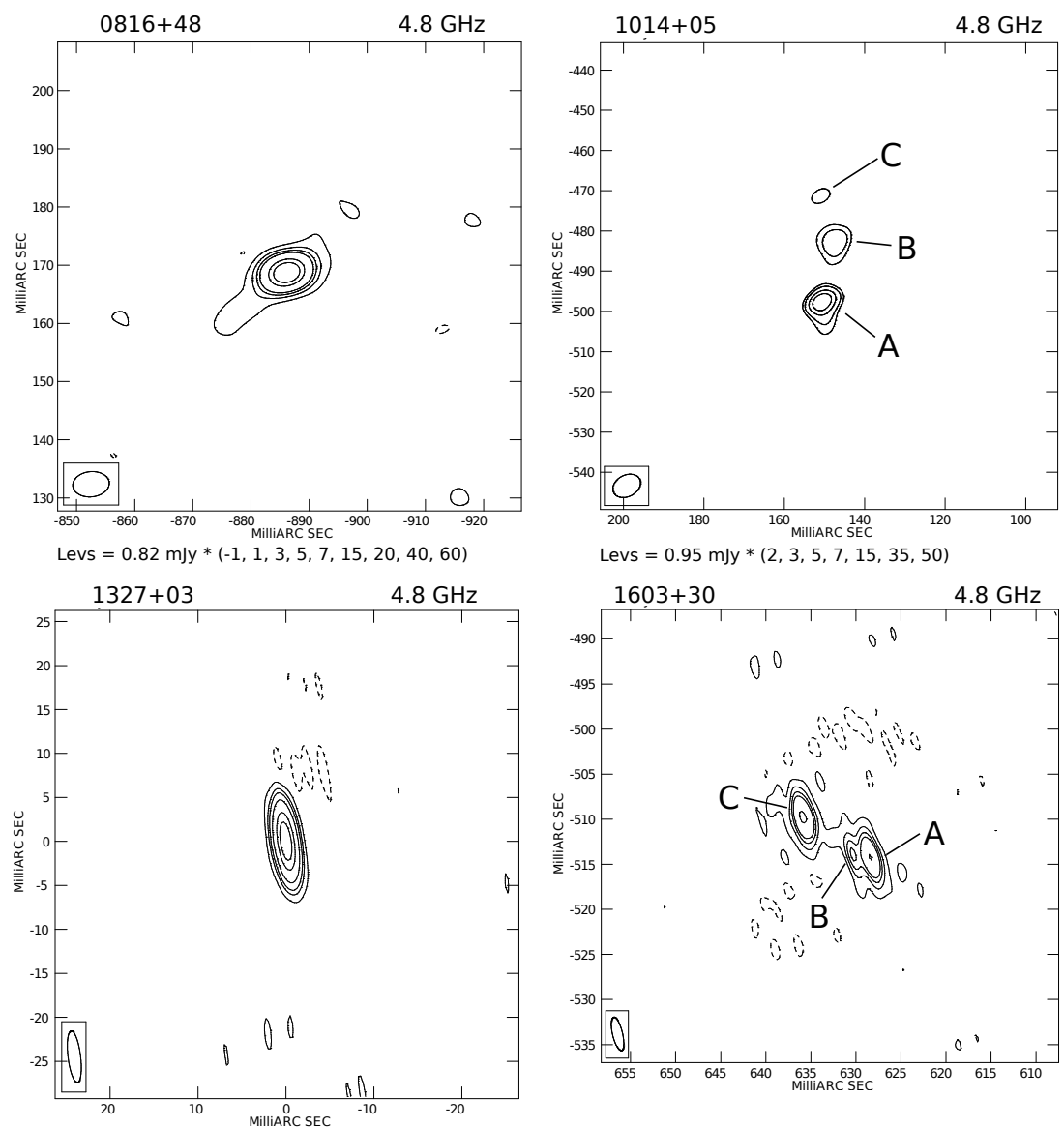

Levs $=1.14 \mathrm{mJy} *(-1,1,3,5,10,25,40)$

Levs $=0.58 \mathrm{mJy} *(-1,1,3,5,7,15,30,50)$

Figure 3: EVN maps at $4.8 \mathrm{GHz}$ of four BAL QSOs. The synthesised beam size is shown in the lower left corner of the map. Levels are 3- $\sigma$ multiples, according to the legend.

J., Holt J., Jiménez Luján F., Radio spectra and polarisation properties of Radio-Loud BAL Quasars, A\&A (in prep.) (2011)

[3] Elvis M., A Structure for Quasars, ApJ, 545, 63 (2000)

[4] Liu Y., Jiang D.R., Wang T.G., Xie F.G., The compact structure of radio-loud broad absorption line quasars, MNRAS, 391, 246 (2008)

[5] Montenegro-Montes F.M., Mack K.-H., Vigotti M., Benn C., Carballo R., González-Serrano J., Holt J., Jiménez Luján F., Radio spectra and polarization properties of radio-loud broad absorption-line quasars, MNRAS, 388, 1853 (2008)

[6] O’Dea C.P, Baum S.A., Constraints on Radio Source Evolution from the Compact Steep Spectrum and GHz Peaked Spectrum Radio Sources, AJ, 113, 148 (1997).

[7] Salerno E., Mack K.-H., Bruni G., Montenegro-Montes F.M., Benn C., Carballo R., González-Serrano J., Holt J., Jiménez Luján F., Multi-frequency study of BAL QSOs radio variability, MNRAS (subm.) (2011)

[8] Sanders D. M., The Evidence for an Evolutionary Connection Between Luminous Infrared Galaxies and Luminous Optically-Selected AGN, ASP conference series, Vol 284 (2002) 